\title{
Endoscopic Ultrasound-Guided Direct Intervention for Solid Pancreatic Tumors
}

\author{
Jimin Han ${ }^{1}$ and Kenneth J. Chang ${ }^{2}$ \\ ${ }^{1}$ Department of Internal Medicine, Catholic University of Daegu School of Medicine, Daegu, Korea, ${ }^{2}$ Division of Gastroenterology and \\ Hepatology, H.H. Chao Comprehensive Digestive Disease Center, University of California, Irvine, Orange, CA, USA
}

Development and use of linear-array echoendoscope and endoscopic ultrasound-guided fine needle aspiration (EUS-FNA) have made endoscopic ultrasound (EUS) more of an interventional procedure than a purely diagnostic procedure. This is a literature review of previously published clinical studies on EUS-guided direct intervention for solid pancreatic tumors, including EUS-guided fine needle injection (EUS-FNI) of antitumor agents, EUS-guided fiducial marker placement, EUS-guided brachytherapy and EUS-guided tumor ablation. Clin Endosc 2017;50:126-137

Key Words: Endosonography; Fine needle injection; Pancreatic neoplasms; Pancreatic neuroendocrine tumor

\section{INTRODUCTION}

With development of linear-array echoendoscope and endoscopic ultrasound-guided fine needle aspiration (EUSFNA), endoscopic ultrasound (EUS) has become more of an interventional procedure than a diagnostic procedure. Nowadays, EUS-guided interventional procedures include not only EUS-FNA, but also EUS-guided drainage of pancreatic fluid collection, EUS-guided celiac plexus neurolysis (EUS-CPN), EUS-guided biliary drainage, EUS-guided gallbladder drainage, EUS-guided pancreatic duct drainage, and EUS-guided drainage for abdominal and pelvic abscesses. ${ }^{1-9}$ Emerging interventional procedures are EUS-guided tumor ablation, EUS-guided vascular intervention, EUS-guided delivery of antitumor agents, EUS-guided fiducial markers placement and brachytherapy, EUS-guided creation of anastomosis, and

Received: March 2, 2017 Revised: March 22, 2017

Accepted: March 23, 2017

Correspondence: Kenneth J. Chang

Division of Gastroenterology and Hepatology, H.H. Chao Comprehensive Digestive Disease Center, University of California, 101 The City Drive South, Building 23, 3rd floor, Irvine, Orange, CA 92868, USA

Tel: +1-714-456-6745, Fax: +1-714-456-7753, E-mail: kchang@uci.edu

(c) This is an Open Access article distributed under the terms of the Creative Commons Attribution Non-Commercial License (http://creativecommons.org/ licenses/by-nc/3.0) which permits unrestricted non-commercial use, distribution, and reproduction in any medium, provided the original work is properly cited.
EUS-guided liver biopsy and portal pressure measurement. ${ }^{10-16}$

The rationale behind performing EUS-guided direct intervention for solid pancreatic tumors is eloquently explained in a literature review on EUS-guided antitumor agent delivery for pancreatic solid tumors: ${ }^{11}$ anatomic location of the pancreas within the abdomen and suboptimal response to systemic antitumor therapy. Access to the pancreas is not easy to obtain since the pancreas is located in the retroperitoneal space and adjacent to hollow viscus, solid organs and major vessels. Even after decades of basic and clinical researches on pancreatic cancer, its prognosis is still dismal and response to systemic chemotherapy less than satisfactory. And another target for EUS-guided direct interventions is pancreatic neuroendocrine tumor. Surgical resection is the treatment of choice and the only chance for cure. However, some patients who are poor surgical candidates or refuse surgical resection have undergone and benefited from EUS-guided tumor ablation. ${ }^{17-23}$ Various methods of EUS-guided direct intervention for solid pancreatic tumors have been utilized in clinical practice with promising results. This article reviews the methods and outcomes of previously published clinical studies on EUS-guided direct intervention for solid pancreatic tumors, namely, EUS-guided fine needle injection (EUS-FNI) of antitumor agents, EUS-guided fiducial marker placement and brachytherapy and EUS-guided tumor ablation. 


\section{ENDOSCOPIC ULTRASOUND- GUIDED FINE NEEDLE INJECTION OF ANTITUMOR AGENTS}

\section{Cytoimplant}

Cytoimplant is an allogeneic mixed lymphocyte culture obtained by co-incubation of peripheral blood mononuclear cells from a tumor patient and a healthy donor. Intratumoral injection of the cytoimplant promotes cytokine production within the tumor itself and results in its regression by activation of immune effector cells. ${ }^{24}$ Before development of EUS-FNI, intratumoral injection had been done through surgery. Chang et al. conducted the first clinical trial which utilized EUS-FNI for injecting antitumor agent directly into a local cancer. ${ }^{24}$ In this phase I clinical trial of eight patients with unresectable pancreatic adenocarcinoma, cytoimplant was delivered into the pancreatic tumor by EUS-FNI. ${ }^{24}$ Under real-time EUS guidance, a 22-gauge needle (GIP/Medi-Globe, Tempe, AZ, USA) was advanced through the pancreatic tumor and a "well" was created with the needle. While the cytoimplant was being injected, the needle was slowly withdrawn simultaneously. A single injection was done. There was no immediate postprocedural complication. The overall median survival of the 8 patients was 13.2 months (range, 4.2-361 months). A multicenter phase II/III trial had been conducted to determine the efficacy of cytoimplant compared with gemcitabine. ${ }^{25}$ However, it was prematurely terminated because response rate and survival were greater for patients randomized to gemcitabine. Cytoimplant is no longer in clinical use because of severe drug-related sepsis.

\section{Gene therapy}

\section{ONYX-015}

ONYX-015 is an E1B-55kD gene-deleted replication-selective adenovirus that preferentially replicates inside and kills malignant tumor cells. ${ }^{26}$ In an open label phase I/II study, 21 patients with locally advanced pancreatic adenocarcinoma or with minimal metastatic disease underwent EUS-FNI of ONYX-015 into the pancreatic tumor. ${ }^{26}$ The patients had eight sessions of EUS-FNI over eight weeks. The final four sessions were done in combination with intravenous gemcitabine. The total volume of ONYX-015 that was injected was one-tenth of the tumor volume, calculated by the three-dimensional measurement of the pancreatic tumor on computed tomography (CT). In the beginning, either gastric or duodenal approach was used. However, the gastric approach was enforced after two cases of duodenal perforation occurred after duodenal approach. A 22-gauge needle (Echotip; Wilson-Cook, Winston-Salem, NC, USA) was utilized for EUS-FNI. Under direct EUS guidance, injections were made in a "fan-like pattern" during withdrawal of the needle. In the beginning of the clinical trial, antimicrobial prophylaxis was administered. After two cases of infections that could have been related to the injection technique, the needle was not pulled all the way into the lumen during the "fan-like pattern" and oral ciprofloxacin was administered before and after the procedure. Although there was no overt acute pancreatitis, hyperlipasemia was observed in two patients and hyperamylasemia in one patient. Two patients had sepsis before institution of oral antimicrobial prophylaxis. Also, two patients had duodenal perforation from the stiff tip of the echoendoscope before mandating transgastric approach. No additional procedure-related complications occurred after refinement of the EUS-FNI protocol. After combination of intratumoral ONYX-015 and intravenous gemcitabine, four patients had partial regression or minor responses and six patients had stable disease. Eleven patients had disease progression or had to discontinue study because of treatment toxicity. Although ONYX-015 itself and combination with gemcitabine were well tolerated, there were four patients with serious complications.

\section{TNFerade}

TNFerade Biologic is a replication-deficient adenovirus type 5 that contains radiation-inducible Egr-1 promoter gene and tumor necrosis factor- $\alpha$ cDNA. ${ }^{27}$ Local injection of TNFerade Biologic allows selective delivery of tumor necrosis factor- $\alpha$ to tumor cells when combined with standard chemoradiotherapy. In a phase I/II study of, Hecht et al. performed intratumoral injection of TNFerade Biologic combined with chemoradiotherapy in patients with locally advanced pancreatic adenocarcinoma. ${ }^{27}$ Either EUS-FNI or percutaneous injection was chosen for each patient. The choice of delivery method was left to the discretion of each participating study site. EUSFNI was performed following a protocol similar to that of a previous study. ${ }^{26}$ Four $0.5 \mathrm{~mL}$ injections into different areas of the pancreatic tumor were made. Twenty seven out of 50 enrolled patients underwent EUS-FNI. Since the study aimed to determine the maximal tolerated dose, safety and feasibility of TNFerade Biologic with chemoradiotherapy, neither technical success nor number of procedure-related complications were specified. There were two cases of acute pancreatitis and both cases had maximum dose of TNFerade Biologics. Delivery method was not specified. Therefore, it could not be determined whether these two cases of acute pancreatitis represent the effect of the disease or the treatment.

\section{BC-819}

BC-819 is a double-stranded DNA plasmid that carries the gene for the diphtheria toxin-A chain under the control 
of the $\mathrm{H} 19$ promoter. ${ }^{28}$ Overexpression of $\mathrm{H} 19$ transcription factors are observed in tumor cells and the presence of these transcription factors triggers expression of diphtheria toxin-A chain. Thus, diphtheria toxin-A expression results in selective tumor cell destruction. In a phase I/II study, the safety, tolerability, pharmacokinetics and efficacy of intratumoral injection of BC-819 were evaluated in patients with unresectable, locally advanced pancreatic adenocarcinoma. ${ }^{28}$ Either percutaneous injection under CT guidance or EUS-FNI was chosen by the principal investigator at each site. Six out of nine enrolled patients underwent EUS-FNI. Either a 21- or 22-gauge needle was utilized. Interestingly, a "clockwise injection site scheme" was used. For the first injection, BC-819 was injected at 12 and 6 oclock of the pancreatic tumor. For the second injection, it was injected at 3 and 9 oclock. For the next injection, it was injected at 6 and 12 oclock. The purpose of clockwise rotation was to allow maximum distribution of BC-819 inside the pancreatic tumor. Injection was administered twice weekly for two weeks. There were no procedure-related complications.

\section{Gemcitabine}

Gemcitabine has been used in treatment of pancreatic adenocarcinoma for more than two decades and its benefit and safety profile are well established. Therefore, it is not surprising that gemcitabine has been chosen in the first clinical investigation of EUS-FNI with standard chemotherapeuctic agents for patients with pancreatic adenocarcinoma. ${ }^{25}$ In this prospective study, 36 patients with locally advanced and metastatic pancreatic adenocarcinoma underwent EUS-FNI with gemcitabine. A 22-gauge fine needle aspiration (FNA) needle (Wilson-Cook Medical Inc., Winston-Salem, NC, USA) was used. The needle tip was placed 0.5 to $1.0 \mathrm{~cm}$ from the tumor edge further from the transducer and injection was done as the needle was withdrawn toward the transducer. About 50\% of the injection was distributed at tumor invasion site and the other $50 \%$ inside the pancreatic tumor. Additional passes and injections were made until there was no more intratumoral spread of gemcitabine. Extent of intratumoral spread, pattern of gemcitabine spread, and homogeneity of gemcitabine spread were visually graded from 1 to 4 with 1 representing the least dispersed pattern and 4 representing a more complete pattern and extent. The concentration of gemcitabine used was $38 \mathrm{mg} / \mathrm{mL}$. Median volume of the injection was $2.5 \mathrm{~mL}$ (range, $0.7-7.0 \mathrm{~mL}$ ). Corresponding intratumoral gemcitabine dose was $95.0 \mathrm{mg}$ (range, 26.6-266 mg). Median number of needle passes was 3 (range, 1-4). In addition to EUS-FNI, 22 patients had undergone subsequent conventional chemoradiotherapy, 10 patients chemotherapy alone, three patients indeterminate therapy, and one patient no therapy. Overall survival at 6 months and 12 months was $78 \%$ and $44 \%$, each respectively. The median overall survival was 10.4 months (range, 3.1-63.9 months). Four out of 20 patients with stage III unresectable pancreatic cancer were downstaged and able to undergo an $\mathrm{R} 0$ resection.

\section{Immunotherapy}

Cancer immunotherapy involves administration of various antigen-presenting cells in order to stimulate tumor recognition and tumor-specific immune response. Dendritic cells are known potent antigen-presenting cells that can induce cytotoxic T lymphocytes. The feasibility, safety, and clinical response of EUS-FNI of immature dendritic cells were investigated in a pilot study. ${ }^{29}$ Seven patients with stage IV pancreatic adenocarcinoma that was refractory to gemcitabine were enrolled. Under real-time EUS guidance, intratumoral injection of immature dendritic cells was made at two to three sites. EUS-FNI was repeated every 7 days and number of EUS-FNI ranged from 2-21. There was no report of procedure-related complications. Two patients had mixed response, two patients had stable disease, and three patients had disease progression. Median survival was 9.9 months (range, 5-21 months).

Although EUS-FNI of antitumor agents seemed promising, clinical studies did not shown noteworthy results in the treatment of locally advanced or unresectable pancreatic cancer. Further development of novel and more effective antitumor agents are necessary.

\section{ENDOSCOPIC ULTRASOUND-GUIDED FIDUCIAL MARKER PLACEMENT AND BRACHYTHERAPY}

\section{EUS-guided fiducial marker placement}

Fiducial markers are inert radiopaque spheres, coils, or cylindricals that are implanted inside or adjacent to the tumor in order to aid image-guided radiation therapy (IGRT). ${ }^{12}$ IGRT allows precisely aimed delivery of radiation to the tumors while minimizing radiation to normal tissues, but requires presence of multiple reference points through which the tumor can be identified and tracked. Before development of EUS-FNA, fiducial markers have been placed either by surgery or percutaneous route under ultrasound or CT guidance.

EUS-guided fiducial marker placement for pancreatic cancer was first reported in a case series of 13 patients with mediastinal and abdominal malignancies. ${ }^{30}$ Seven patients were treated for pancreatic cancer; five had unresectable adenocarcinoma, one a recurrent adenocarcinoma after pancreaticoduodnectomy, and one a plasmocytoma. A 19-gauge FNA needle (MEDI-Globe, Achenmühle, Germany, or Sonotip II, Wilson-Cook, Winston-Salem, NC, USA) was used. The 
fiducials were gold cylinders with a length of 3 or $5 \mathrm{~mm}$ and a diameter of $0.8 \mathrm{~mm}$ (Best Medical International Inc., Springfield, VA, USA). After insertion of the FNA needle into the tumor, the stylet was removed and a fiducial was manually placed into the needle. The stylet was used to push the fiducial through the needle lumen into the tumor. This was repeated to place three to six fiducials. Fluoroscopy was also used to verify location of fiducial placement. EUS-guided fiducial marker placement was successful in 11 of 13 patients (84.6\%). In two patients, the FNA needle could not be inserted into the tumor: one patient with gastric outlet obstruction from the pancreatic head cancer and the other patient with tumor located posterior to the aorta. There was no immediate procedure-related complication.

Further studies of EUS-guided fiducial marker placement followed. A prospective case series ${ }^{31}$ reported a single-center experience of EUS-guided fiducial placement in 57 patients with unresectable pancreatic cancer. A 19-gauge FNA needle (Echotip Ultra 19; Cook Endoscopy, Winston-Salem, NC, USA) was used. Gold fiducials with length of $2.5 \mathrm{~mm}$ and diameter of $0.8 \mathrm{~mm}$ (Alpha-Omega Services, Bellflower, CA, USA) were used. Fluoroscopy was not used for verification of fiducial placement. Before the procedure, the FNA needle was preloaded with the fiducials by removal of the stylet and manual back-loading of 2 fiducials into the tip of the needle. For the first 14 patients, sterile lubricant was utilized to separate and maintain the position within the needle. For the subsequent 43 patients, sterile bone wax was used instead of the jelly. Fiducials were pushed out of the needle by using a stylet for the first five patients. But, for the subsequent 52 patients, the fiducials were deployed by injecting 1 to $2 \mathrm{~mL}$ of sterile water through the needle after stylet removal. A minimum of two fiducials were inserted. The procedure was successful in 50 of 57 patients (88\%). Fiducial placement was not attempted in one patient because of gastric outlet obstruction from pancreatic head tumor. There were minor complications in three patients. Needle malfunction without any effects on the patients occurred in two patients. Minor bleeding developed limiting the number of fiducials placed. However, the number of fiducials was adequate for IGRT. This study was significant because it introduced a new fiducial insertion technique that did not require use of a stylet or fluoroscopic guidance.

A prospective study evaluated the safety, feasibility, and limitations of EUS-guided placement of fiducials for locally advanced and recurrent pancreatic cancer. ${ }^{32} \mathrm{~A}$ total of 51 patients were included. A 19-gauge FNA needle (Echotip; Cook Endoscopy, Winston-Salem, NC, USA) was used. In this study, a $0.8 \times 5.0 \mathrm{~mm}$ gold cylindrical fiducial marker (Alpha-Omega Services, Bellflower, CA, USA) was back-loaded into the needle tip and sealed into place with sterile bone wax. After the needle was inserted into the tumor under EUS guidance, the fiducial was deployed by pushing of the stylet. Only EUS guidance was used. In the beginning of the study, two fiducials were placed into the tumor. Later, an additional fiducial was implanted in the left lobe of the liver to avoid additional EUS-guided placement in case of possible fiducial migration. EUS-guided fiducial placement was successful in 46 of 51 patients (90\%). EUS-guided fiducial placement failed in 4 patients (8\%). These patients had recurrent cancer after pancreaticoduodenectomy. Migration of fiducial occurred in 3 patients (7\%). There was one patient who had mild acute pancreatitis after undergoing simultaneous EUS-CPN and fiducial placement. After 2 days of hospitalization and treatment, the patient was discharged in stable condition.

A case series of nine patients with locally advanced pancreatic cancer or a positive retroperitoneal R1 resection margin at pancreaticoduodenectomy showed technical feasibility and safety of EUS-guided fiducial placement..$^{33}$ A 19-gauge FNA needle with a stylet in place was used. After withdrawal of the stylet by $2 \mathrm{~cm}$, one gold fiducial (Best Medical International Inc., Springfield, VA, USA) was back-loaded into the FNA needle and sealed with sterile bone wax. The length of a fiducial was $3 \mathrm{~mm}$ and its diameter was $0.8 \mathrm{~mm}$. Each and every fiducial was deployed by advancing the stylet under EUS and fluoroscopic guidance. For patients with locally advanced pancreatic cancer, five fiducials were deployed into the pancreatic tumor. For patients with positive resection margin, 3 fiducials were deployed at the resection margin. When the fiducials could not be deployed by advancing the stylet (e.g., in patients with pancreatic uncinate or head tumors), the stylet was removed from the FNA needle and flushing of $1-2 \mathrm{~mL}$ of normal saline was done instead. Two patients with pancreatic uncinate or head tumors had fiducial deployment by using saline flushing. EUS-guided fiducial placement had technical success rate of $100 \%$ and no procedure-related complications. The mean duration of EUS-guided fiducial placement was 9.5 minutes (range, 7-12 minutes). For two patients who had simultaneous EUS-FNA and celiac plexus neurolysis (CPN), the mean duration of the procedure was 28 minutes (range, 24-32 minutes). Normal saline has been used for the first time to deploy the fiducials. Although the number of patients was small, it seemed to be safe to perform three EUS-guided interventions-FNA, CPN, and fiducial placement-during single session.

A retrospective, single-center study compared two types of commercially available fiducials after EUS-guided placement. ${ }^{34}$ In particular, technical success, complications, visibility, and migration rate of the fiducials were compared. Traditional fiducials are $5 \mathrm{~mm}$ in length and $0.8 \mathrm{~mm}$ in diameter and requires a 19-gauge FNA needle for deployment. 
On the other hand, Visicoil fiducials are $10 \mathrm{~mm}$ in length and $0.35 \mathrm{~mm}$ in diameter and can be loaded into a 22-gauge FNA needle. Theoretically, Visicoil fiducials would be easier to implant in pancreatic head, but their visibility could be similar or worse than that of traditional fiducials. In this study, 39 patients with locally advanced pancreatic cancer were included and had 109 fiducials placed under EUS-guidance. An FNA needle (Echotip; Cook Endoscopy, Winston-Salem, NC, USA) with the stylet in place was used. The stylet was withdrawn 2-3 $\mathrm{cm}$ and the FNA needle was back-loaded with single fiducial marker. The needle tip was sealed with sterile bone wax. After the needle was inserted into the pancreatic mass, the fiducial was deployed by advancing the stylet under EUS guidance. From a total of 109 fiducials, 77 were traditional fiducials and 26 Visicoil fiducials. There was no significant difference of number of fiducials placed per patient. No procedure-related complications were reported. As expected, visibility of traditional fiducials was significantly better than that of thinner Visicoil fiducials. Fiducial migration rate was not significantly different. Authors stated that their preference regarding EUS-guided fiducial placement is to use traditional fiducials when possible. However, changing to Visicoil fiducials seemed advisable if placement of traditional fiducials is technically difficult.

Since only one fiducial could be deployed at single FNA needle pass, removal of the FNA needle and manual loading of each and every fiducial were required for delivery of multiple fiducials. And there had been no dedicated accessory for EUS-guided fiducial placement. A new multi-fiducial delivery system (Cook Medical Inc., Bloomington, IN, USA) had been developed and evaluated in a live porcine model. ${ }^{35}$ This system consists of a stainless steel 22-gauge delivery needle that is preloaded with 4 gold fiducials. The fiducial is $5 \mathrm{~mm}$ in length and $0.43 \mathrm{~mm}$ in diameter. In order to prevent the preloaded fiducials from premature deployment, there is a small waist in the needle channel distal to the fiducials. Fiducial is deployed by gradual advancement of the stylet through the waist and a 'release' is felt as each fiducial is deployed. Technical success in the porcine model was $95.6 \%$ and time duration for deployment of 4 fiducials was less than 1 minute. This new multi-fiducial delivery system is being evaluated in an ongoing clinical study. ${ }^{36}$

A retrospective cohort study evaluated achievement of ideal fiducial geometry and success of fiducial tracking in patients with advanced pancreatic cancer who underwent either EUS-guided or surgery-assisted fiducial placement. ${ }^{37}$ Ideal fiducial geometry is defined as placement of at least three fiducials with a minimum interfiducial distance of greater than $2 \mathrm{~cm}$, minimum interfiducial angle of greater than 15 degrees, and noncollinear placement in the imaging plane. ${ }^{37}$ In this study, a 19-gauge FNA needle with the stylet in place was used. A gold fiducial was back-loaded into the FNA needle after withdrawal of the stylet by $2 \mathrm{~cm}$ and sealed with sterile bone wax. The fiducial was deployed into the pancreatic tumor by advancing the stylet. The analysis of 77 patients showed that EUS-guided placement resulted in successful fiducial tracking despite lower proportion of patients with ideal fiducial geometry. ${ }^{37}$ The proportion of patients with ideal fiducial geometry was significantly lower for EUS-guided fiducial placement than surgery-assisted placement (18\% vs. $47 \%, p=0.0011)$. However, success rate of fiducial tracking was not different ( $82 \%$ vs. $90 \%$ ). Of note, there were five procedure-related complications in patients with EUS-guided fiducial placement; one patient had mild acute pancreatitis that required admission for two days. Three patients had abdominal pain and one had vomiting. Except for the patient with mild acute pancreatitis, all the other patients improved after management in the recovery room and were discharged.

Another study assessed the safety and technical feasibility of EUS-guided fiducial placement in 29 patients with pancreatic cancer and three hepatocellular carcinoma. ${ }^{38}$ All the patients had been administered peri-procedural antibiotics prophylaxis for one day. A 19-gauge FNA needle (Echotip; Cook Endoscopy, Winston-Salem, NC, USA) was used. The gold fiducial was $3 \mathrm{~mm}$ in length and $0.8 \mathrm{~mm}$ in diameter (CIVCO Medical Solutions, Orange City, IA, USA). After withdrawing the stylet by $7-8 \mathrm{~mm}$, the fiducial was back-loaded into the needle tip and sealed with sterile bone wax. The fiducial was deployed by advancing the stylet. Fluoroscopy was not used to verify the fiducial placement. EUS-guided fiducial placement was successful in all the patients. The mean number of fiducials placed per patient was 2.9 (range, 2-3) and fiducial migration rate was $3.1 \%$. One patient with pancreatic head cancer had mild acute pancreatitis requiring admission for two days. Five patients had EUS-FNA and fiducial placement during a single session. None had procedure-related complication. ${ }^{38}$

Dávila Fajardo et al. evaluated the safety and feasibility of EUS-guided fiducial placement by using a 22-gauge needle in 23 patients with pancreatic cancer. ${ }^{39}$ In this study, Visicoil (Core Oncology, Santa Barbara, CA, USA) and the Gold Anchor fiducial (Naslund Medical AB, Huddinge, Sweden) were used. The Gold Anchor fiducial has length of $10 \mathrm{~mm}$ and diameter of $0.28 \mathrm{~mm}$. Prophylactic antibiotics were not routinely administered. A fiducial was back-loaded into the tip of the FNA needle and sealed with sterile bone wax. Fluoroscopy was used if it was available. Because this was not a randomized study, 17 patients had Visicoil fiducials, four had Gold Anchor fiducials, and two had both types. All the patients had at least two fiducials implanted in the pancreatic tumor (range, 2-4). The technical success rate was $100 \%$. The fiducial migration 
rate was $9.5 \%$. IGRT was successfully done in $95.2 \%$ of the patients who received radiation therapy. One patient had minor bleeding at the site of needle insertion. The procedure was terminated after placement of two fiducials.

A retrospective study of 514 patients with gastrointestinal malignancies who underwent EUS-guided fiducial placement without fluoroscopy demonstrated its technical feasibility and safety. ${ }^{40}$ There were 188 patients with pancreatic cancer in this study and 510 fiducials were placed in these patients. ${ }^{40}$ Either a 19-gauge or 22-gauge EUS-FNA needle (Cook Endoscopy, Winston-Salem, NC, USA) was utilized. A gold fiducial (Visicoil; RadioMed Inc., Tyngsborough, MA, USA) with length of $10 \mathrm{~mm}$ and two different diameters $(0.75 \mathrm{~mm}$ or $0.35 \mathrm{~mm})$ was used. The stylet was withdrawn by $7-8 \mathrm{~mm}$. Then, the fiducial was back-loaded into the needle tip and sealed with sterile bone wax. The fiducial was deployed by simultaneous retraction of the needle and advance of the stylet. All the fiducials were implanted under EUS guidance alone, without fluoroscopy. A 22-gauge needle was used in 150 patients and a 19-gauge needle was used in 37 patients. The technical success was $99.5 \%$. In one patient, no fiducial was placed due to the intervening blood vessels. The fiducial migration rate was $0.4 \%$. Seven patients had minor bleeding.

Most of the published studies on EUS-guided fiducial placement for pancreatic tumors included pancreatic adenocarcinoma prior to IGRT. There has been a case series in which EUS-guided fiducial marker placement was performed in two patients with pancreatic neuroendocrine tumor for preoperative localization. ${ }^{41}$ Instead of EUS-guided tattooing, two patients with a $7 \mathrm{~mm}$ and a $9 \mathrm{~mm}$ pancreatic neuroendocrine tumors underwent EUS-guided fiducial placement. Tumors were located in the uncinate process and neck of the pancreas, respectively. In both patients, two Visicoil fiducials (Core Oncology, Santa Barbara, CA, USA) were placed either within or adjacent to the tumors by using a 22-gauge FNA needle (Echotip; Cook Endoscopy, Winston-Salem, NC, USA). Then, the patients underwent parenchymal-sparing pancreatic surgery. In all the patients, the fiducials were well-visualized by intraoperative ultrasound, and the surgical resection was successful. There were no procedure-related complications. In addition, there was no evidence of pancreatitis in the resected pancreas. Therefore, EUS-guided fiducial placement may be useful for rapid, accurate localization and parenchymal-sparing resection of small pancreatic tumors.

Both prospective and retrospective studies demonstrated safety and technical feasibility of EUS-guided fiducial placement in solid pancreatic tumors. Moreover, EUS-guided fiducial placement can be done without the use of fluoroscopy. However, further refinements in fiducial deployment are needed and the new multi-fiducial delivery system seems promising.

\section{EUS-guided brachytherapy}

Radioactive seeds used for brachytherapy include iodine-125, iridium-192, and palladium-103. ${ }^{12}$ Among these, the most commonly used radioactive seed for pancreatic cancer is iodine-125; iodine- 125 has a longer half-life of 59.7 days and is deemed more appropriate for treatment of rapidly growing tumors such as pancreatic cancer. Traditionally, radioactive seeds have been implanted during open laparotomy or by image guidance. With development of interventional EUS, EUS-guided brachytherapy had been increasingly utilized. Its advantages are the precise localization of radiation in the tumor itself and the minimization of damage to the surrounding normal tissue.

A pilot study of EUS-guided brachytherapy in 15 patients with advanced pancreatic study was reported in $2006 .{ }^{42}$ Before the implantation, the tumor volumes and number of implants required were calculated. Then, distribution plan maps were drawn and the distance and direction of every target site from the center of the pancreatic tumor were determined. A 19-gauge FNA needle (Wilson-Cook Medical Inc., Winston-Salem, NC, USA) was used for iodine-125 seeds implantation. After the needle insertion into the pancreatic tumor, the stylet was removed and a radioactive seed was inserted into the lumen of the needle. The seed was deployed by advancing the stylet. The mean duration of time for EUS-guided implantation was 28 minutes. The mean number of needle passes per procedure was 14 (range, 6-21). The mean number of radioactive seeds implanted was 22 per patient (range, 1130). None of the patients had chemotherapy after EUS-guided brachytherapy. Three patients had acute pancreatitis and two out of the three patients developed pseudocysts. The overall response rate was $80 \%$. Partial response was seen in $26.7 \%$, minor response in $20 \%$, and stable disease in $33 \%$. The median duration of the partial response was 4.5 months. Improvement in performance status and pain was seen in $33 \%$. The overall median survival was 10.6 months (range, 4.2-25 months). At two years after brachytherapy, $6.7 \%$ of the patients survived.

Another prospective pilot study of EUS-guided brachytherapy using iodine- 125 seeds in 22 patients with advanced pancreatic cancer demonstrated that it was technically successful and safe. ${ }^{43}$ Although brachytherapy seemed to relieve abdominal pain, it did not affect survival. All the patients received gemcitabine-based 5-fluorouracil chemotherapy one week after brachytherapy. The radioactive seed was 4.5 $\mathrm{mm}$ in length and $0.8 \mathrm{~mm}$ in diameter. It had a radioactivity half-life of 60.1 days, a mean photon energy of $27-35 \mathrm{KeV}$, a human tissue penetration distance of $1.7 \mathrm{~cm}$, an initial dose rate of $7 \mathrm{cGy} / \mathrm{h}$, and a mean radioactivity of $0.694 \pm 0.021$ 
$\mathrm{mCi}(25.6 \mathrm{MBq})$. Before EUS-guided brachytherapy, the total volume of each tumor was calculated by using the CT image and the expected number of implanted seeds was calculated by using the modified Cevec formula. For maximal radiation effect, the number of seeds implanted was $15 \%$ more than calculated. EUS-guided implantation of iodine-125 seeds (Xinke Pharmaceutical Co., Ltd., Shanghai, China) was successful in all the patients. A median number of tem radioactive seeds (range, 5-30) was implanted per procedure. A mean puncture frequency was 3.2 times per procedure. Nineteen patients had only one implantation. Two patients had two implantations and the remaining one patient had three implantations within three months. There was no procedure-related complication. The median follow-up duration was 9.3 months and the median survival time was 9.0 months. During the 4 -week period, partial response was noted in $13.6 \%$ and stable disease in $45.5 \%$. All the patients died within two years. The visual analog scale pain score decreased from 5.1 to $1.7(p<0.01)$ at one week after brachytherapy, but increased to 3.5 at one month $(p<0.05)$.

An interesting pilot study evaluated the result of EUS-guided brachytherapy combined with intratumoral implants for sustained delivery of 5-fluorouracil in eight patients with advanced pancreatic cancer. ${ }^{44}$ This procedure was named EUS-guided interstitial chemoradiation. Before implantation, the tumor volume and required seed number were calculated. The total number of the chemotherapeutic implants was determined to be twice as many as the number of seeds. Then distribution plan maps were drawn, and the distance and direction of every target site from the center of the pancreatic tumor were determined. A 19-gauge FNA needle with a stylet was used for seeds and implant deployment. In each patient, a mean of 19 iodine-125 radioactive seeds (China Institute of Atomic Energy, Beijing, China) and 36 intratumoral implants for sustained delivery of 5fluorouracil were implanted. The mean number of needle passes per procedure was 13 (range, 8-16). The procedure was successful in all the patients. The mean time duration of the procedure was 35 minutes. The mean total implanted radioactive activity was $13.68 \mathrm{mCi}$ and the mean total dose of intratumoral 5fluorouracil was $3.6 \mathrm{~g}$. There was no procedure-related complication.

\section{ENDOSCOPIC ULTRASOUND-GUIDED TUMOR ABLATION}

\section{Ethanol ablation}

EUS-guided ethanol ablation of solid pancreatic tumors was first reported in 2006. ${ }^{17}$ A 78-year-old woman with repeated hypoglycemia had been diagnosed with insulinoma. Because of her poor condition, severe complications during hypogly- cemic episodes and refusal of surgical resection, the patient had been treated with EUS-guided ethanol ablation. A total of $8 \mathrm{~mL} 95 \%$ alcohol, divided into 4 portions of $2 \mathrm{~mL}$, was injected into the pancreatic tumor using a 22-gauge needle (Vilman-Hancke-Needle; GIP Medizin Technik, Grassau, Germany). The needle position was changed and injection repeated until the entire tumor appeared hyperechoic. Although there were no further hypoglycemic episodes, the patient had mild acute pancreatitis after EUS-guided ethanol ablation which resolved after three days. The authors attributed the development of mild acute pancreatitis to the large volume of ethanol injected. No tumor recurrence was noted up to 34 months of follow-up.

More case reports and case series followed the first report. Muscatiello et al. ${ }^{18}$ reported a patient with two pancreatic neuroendocrine tumors, who had undergone EUS-guided ethanol ablation. The patient refused surgical resection and underwent the procedure. Two sessions of ethanol ablation were done. Less than $2 \mathrm{~mL}$ of $40 \%$ ethanol was injected during each session. Although the first session was completed without complications, the second session was complicated by ethanol effusion and development of a small pancreatic necrotic lesion, requiring laparoscopic necrosectomy. Somatostatin receptor scintigraphy and measurement of serum vasoactive intestinal peptide and chromogranin A done two months later were normal. Deprez et al. ${ }^{19}$ reported a case of insulinoma in the pancreatic head with symptomatic hypoglycemia which was treated with EUS-guided ethanol ablation. The patient was a 78-year-old woman and could not undergo pancreaticoduodenectomy because of poor general condition. For ablation, $3.5 \mathrm{~mL}$ of $98 \%$ ethanol was injected into the pancreatic tumor. There was no recurrence of hypoglycemia for more than two years after the ablation. However, there was a case of mild and asymptomatic elevation of pancreatic enzymes for two days and another case of duodenal hematoma and ulceration that were treated conservatively. Vleggaar et al. ${ }^{20}$ reported a case of insulinoma with symptomatic hypoglycemia which was successfully treated with EUS-guided ethanol ablation. The patient was an 82-year-old woman with heart failure and a poor surgical candidate. For the procedure, $0.3 \mathrm{~mL}$ of $96 \%$ ethanol was injected into the tumor in the pancreatic body using a 25-gauge needle (EchoTip Ultra; Cook, Limerick, Ireland). There were no symptoms of hypoglycemia up to six month after the ablation.

In a case series of eight patients with insulinomas, ${ }^{21}$ five patients had been treated with EUS-guided ethanol ablation and the remaining three with intraoperative ultrasound-guided ethanol ablation. For the five patients who had EUS-guided ethanol ablation, surgical resection was not done because of the following reasons: wish to avoid pancreaticoduodenecto- 
my $(n=2)$, significant comorbidities $(n=2)$, and history of multiple abdominal operations $(n=1)$. All the patients had experienced frequent and severe hypoglycemic episodes for a mean of 5.6 years (range, 0.1-20 years). Either a 22- or 25-gauge needle (Wilson-Cook Medical Inc., Winston-Salem, NC, USA) was used for EUS-guided ethanol ablation. Four patients had two treatment sessions and one had three treatment sessions. Number of ethanol injections per session ranged from two to six. Concentration of ethanol used varied from $95 \%$ to $99 \%$. A mean ethanol volume of $0.8 \mathrm{~mL}$ (range, $0.12-3.0 \mathrm{~mL}$ ) per session was injected into the pancreatic tumor. Injections were done in 0.01 to $0.1 \mathrm{~mL}$ at a time and repeated at the same site until a hyperechoic change was visible inside the tumor, but not beyond the tumor border. Additional injections were done as the needle was being withdrawn and until almost complete removal of the needle. Then, the needle was held in place for approximately one minute to avoid tracking of ethanol into the nearby organs. When additional needle passes were made, care was taken to avoid the previous needle tracts. There was no procedure-related complication during or after EUS-guided ethanol ablation. Another case series of four patients with insulinoma who declined surgical resection and underwent EUS-guided ethanol ablation demonstrated successful ablation in single session, no procedure-related complication, and resolution of hypoglycemic symptoms in all the patients. ${ }^{22}$ Also, there was no symptom recurrence during the follow-up up to six months. For all the patients, a 22-gauge needle (Wilson-Cook Medical Inc., Winston-Salem, NC, USA) and $95 \%$ ethanol was used. The volume of ethanol injection was calculated by using the major and minor axes of the tumor. When the tumor was located close to a vessel or the pancreatic duct, the volume of the injected ethanol was reduced to one half or one third of the calculated volume. Volume of the injected ethanol ranged from 0.25 to $0.5 \mathrm{~mL}$. In a pilot study, ${ }^{23}$ 11 patients with 14 pancreatic tumors (ten non-functioning neuroendocrine tumors and four insulinomas) underwent successful EUS-guided ethanol ablation. A 22-gauge needle (EchoTip Ultra or ProCore; Cook Endoscopy, Winston Salem, NC, USA) and 99\% ethanol was used for injection. Unlike previous studies, the optimal volume of ethanol to be injected was calculated by computer estimation of areas on each axial image using an EUS software. In this study, volume of ethanol injection ranged from 0.5 to $3.8 \mathrm{~mL}$. There were three cases of mild pancreatitis. Response rate after single treatment session which was assessed with cross-sectional imaging was 53.8\%. Response rate after multiple sessions in three tumors with residual viable tissue was $61.5 \%$. There was no disease progression during follow-up which ranged from 100 to 730 days.

Although published studies on EUS-guided ethanol ablation of pancreatic neuroendocrine tumors showed high success rate and low procedure-related complication rate, this procedure should be reserved for patients who are poor surgical candidates or who refuse curative resection. Since previous studies comprise mostly of case reports, case series and pilot studies, multicenter controlled studies are needed to prove efficacy of this procedure. Moreover, further refinements are necessary regarding the volume of ethanol to be injected, the optimal concentration of ethanol, the techniques of EUS-guided ethanol ablation, and the assessment of the treatment response.

In addition to pancreatic neuroendocrine tumors, EUS-guided ethanol ablation had been performed in combination with EUS-CPN for pain control and possible delay in disease progression for advanced pancreatic cancer with opioid refractory pain. The first case ${ }^{45}$ was a 75 year-old male with stage IV pancreatic adenocarcinoma and abdominal pain that was 9 out of 10 according to the visual analog scale. EUS-CPN was performed first, and, then, $40 \mathrm{~mL}$ of $95 \%$ ethanol was injected into the tumor. There was no immediate complication after the procedure except for mild diarrhea and fever in the following days. Complete pain relief was achieved three days after the treatment and lasted for ten weeks. Pain was controlled with paracetamol for 20 weeks until death at 30 weeks after the treatment. Later, the same authors reported a retrospective analysis of prospectively collected data on EUS-guided ethanol ablation combined with EUSCPN versus EUS-CPN alone in pancreatic cancer patients. ${ }^{46}$ A total of 123 unresectable pancreatic cancer patients were included. Fifty-eight patients treated with EUS-CPN and 65 with the combined approach were compared. A 22-gauge needle (Cook Medical Inc., Bloomington, IN, USA) was used for both EUS-guided ethanol ablation and EUS-CPN. For EUS-CPN, $10 \mathrm{~mL}$ of $2 \%$ lidocaine and $20 \mathrm{~mL}$ of $95 \%$ ethanol were used. For EUS-guided ethanol ablation, a calculated volume of $95 \%$ ethanol ( $75 \%$ of the pancreatic tumor volume) was injected until the hyperechoic blush extended to the margin of the tumor. The needle tip was maintained within the pancreatic tumor during the procedure. There were no significant differences in the pre-procedural visual analogue scale score (7/10 in EUS-CPN group vs. 7/10 in the combined approach group, $p=0.8$ ) and the maximum diameter of the tumor ( $38 \mathrm{~mm}$ vs. $43 \mathrm{~mm}, p=0.4$ ). The combined treatment group had increased pain relief and complete pain response rate ( $p=0.005$ and $p=0.003$, respectively). Also, the combined treatment group had a longer median duration of pain relief ( 18 weeks vs. 10 weeks, $p=0.004$ ). Multivariate analysis showed that initial visual analogue scale score and combined treatment were significantly associated to pain relief. There were no severe treatment-related adverse events. There was a trend for longer median overall survival in 
the combined treatment group (8.3 months vs. 6.5 months, $p=0.05)$. EUS-guided ethanol ablation combined with EUS$\mathrm{CPN}$ in patients with advanced pancreatic cancer seems to be superior to EUS-CPN alone regarding pain control and overall survival. Even though the results are very promising, prospective randomized trials are necessary in order to confirm the results of this retrospective study.

\section{Radiofrequency ablation}

Radiofrequency ablation (RFA) has been used for the treatment of primary and metastatic liver cancers. Recently, there have been clinical studies investigating its role in EUS-guided treatment of pancreatic tumors. The first human pilot safety and feasibility study using a novel EUS-guided radiofrequency catheter was published in $2015 .{ }^{47}$ This prospective multicenter study enrolled eight patients: six with pancreatic cystic neoplasm and two with neuroendocrine tumors in the pancreatic head. The mean diameter of pancreatic neuroendocrine tumors was $27.5 \mathrm{~mm}$. Those two patients with pancreatic neuroendocrine tumors were poor surgical candidates. A novel monopolar radiofrequency catheter (Habib ${ }^{\text {Txt }}$ EUSRFA catheter; EMcision Ltd., London, United Kingdom) was inserted through a 19-gauge FNA needle. This catheter is a 1-French wire $(0.33 \mathrm{~mm})$ and has a working length of $190 \mathrm{~cm}$. For EUS-guided radiofrequency ablation (EUS-RFA), an FNA needle with stylet in place was first placed in the deepest part of the tumor. Then, the stylet was removed and a RFA catheter was pushed into the FNA needle until resistance is met. The FNA needle was slowly withdrawn by $3 \mathrm{~cm}$ in order to be separate from the active part of the RFA catheter. Fluoroscopy was used for visualization of the RFA catheter. The energy was applied for 90 seconds at $20 \mathrm{~W}$. A change in vascularity and central necrosis area of $15 \mathrm{~mm}$ were noted after EUS-RFA in each patient, respectively. Six and four RFA applications were done per session and one and two sessions were performed in total, each repsectively. Technical success of EUS-RFA was $100 \%$. There was no major complication within 48 hours of the procedure. Both patients were alive at the time of publication.

Another study evaluated the feasibility of EUS-RFA for treatment of symptomatic insulinoma by using a different EUS-RFA needle electrode. ${ }^{48}$ Three patients with symptomatic pancreatic insulinoma who refused surgical resection underwent EUS-RFA in an observational human case series. The EUS-RFA system used (STARmed Co., Ltd., Seoul, Korea) was comprised of a prototype 19-gauge needle electrode, a generator, and an internal cooling system. The length of the uninsulated, conical tip of the needle electrode was $10 \mathrm{~mm}$. Under EUS guidance, the needle electrode was passed into the pancreatic tumor at the far end within the margin. The energy delivery was set at $50 \mathrm{~W}$. In 10 to 15 seconds, a coagulation necrosis area of 10 to $12 \times 5 \mathrm{~mm}$ was created. Further ablation was done by withdrawal of the electrode to more proximal site, through the same needle tract. Additional passes were made by using a fanning technique in order to ablate different areas of the same tumor. Appearance of echogenic bubbles around the needle tip indicated completion of RFA at the site. Of note, the most technically challenging area of the tumor was ablated first since visual artifacts after RFA may hinder accurate targeting. EUS-RFA was technically successful in all the patients. During the follow-up at 11 to 12 months, all the five patients had remained symptom free. There were no procedure-related complications.

EUS-RFA has been utilized treat unresectable pancreatic cancer in addition to pancreatic neuroendocrine tumors. In a cohort of patients with local progression of advanced pancreatic cancer after neoadjuvant therapy, feasibility and safety of EUS-guided cryothermal ablation was prospectively evaluated. ${ }^{49}$ The cryotherm probe is a flexible bipolar radiofrequency probe combined with cryogenic cooling. The distal tip of the probe is sharp and stiff; it can penetrate the gastrointestinal wall and pancreatic parenchyma. A total of 22 patients were enrolled. $18 \mathrm{~W}$ was applied and pressure for cooling was 650 pounds per square inch (psi). Application time was dependent on tumor size. Cryothermal ablation was technically successful in 16 patients (72.8\%). The probe could not penetrate the tumor in six patients because of stiffness of the gastrointestinal wall and the tumor itself. There were three patients with asymptomatic hyperamylasemia, three patients with post-treatment abdominal pain, and one patient with minor duodenal bleeding that was treated with endoscopic hemostatic clip placement. The median post-treatment survival was six months (range, 1-12 months). EUS-guided cryothermal ablation seemed feasible and safe. However, technical improvement of the cryothermal probe and randomized, controlled trials seemed necessary to demonstrate survival benefit of EUS-guided cryothermal ablation in patients with advanced pancreatic cancer.

The feasibility and safety of another EUS-RFA needle probe was evaluated in a preliminary study of six patients with unresectable pancreatic cancer. ${ }^{50}$ The tumors were located in pancreatic head $(n=4)$ and body $(n=2)$. The median diameter of the pancreatic tumors was $3.8 \mathrm{~cm}$ (range, 3-9 cm). An 18-gauge EUS-RFA needle electrode and a radiofrequency generator (STARmed Co., Ltd., Goyang, Korea) were used for the procedure. The length of the uninsulated tip of the RFA electrode was $10 \mathrm{~mm}$. Under real-time EUS guidance, the RFA electrode was inserted into the mass, the energy was delivered 20 to $50 \mathrm{~W}$ for ten seconds. The energy delivery was repeated until the hyperechoic area around the electrode tip covered the tumor. The technical success of EUS-RFA was 100\%. Ex- 
cept for two patients with mild abdominal pain, there were no procedure-related complications. Because of the study design, overall survival could not be assessed.

\section{Photodynamic therapy}

EUS-guided photodynamic therapy (EUS-PDT) of the pancreas has been done mostly in animal studies. ${ }^{51,52}$ The first clinical experience with EUS-PDT in pancreatic cancer has been published in $2015 .{ }^{53}$ Four patients with locally advanced pancreaticobiliary cancers were included. There was one patient with pancreatic tail cancer who had localized tumor progression despite chemoradiotherapy. The tumor diameter was $3.1 \mathrm{~cm}$. For EUS-PDT, a chlorin e6 derivative (Photolon; Belmedpreparaty, Minsk, Republic of Belarus) and a flexible laser-light catheter (PhotoGlow; South Yarmouth, MA, USA) were used. Diffuser length of the catheter was between 1 and $2 \mathrm{~cm}$ and it was $2 \mathrm{~cm}$ for the patient with pancreatic cancer. The photosensitizer was administered intravenously at a dose of $2.5 \mathrm{mg} / \mathrm{kg}$ three hours before the procedure. Before the procedure, the laser-light catheter was preloaded inside a 19-gauge FNA needle (Cook Endoscopy, Winston-Salem, NC, USA). And the catheter was advanced until it was placed within the needle tip and secured with a locking device. The FNA needle preloaded with the laser-light catheter was inserted into the tumor under real-time EUS guidance. Then, the needle was withdrawn $2 \mathrm{~cm}$ while the catheter was advanced in order to be directly contact ing with the tumor. The position of the catheter was secured with the locking device. Photoactivation at a $660-\mathrm{nm}$ wavelength was performed (UPLFDT; LEMT Research \& Development Private Unitary Enterprise, Minsk, Republic of Belarus). The light irradiation was done for 330 seconds and energy dose was $100 \mathrm{~J} / \mathrm{cm}$ of diffuser length. Two laser-light deliveries were done per session. The duration of procedure was 29 minutes. It was technically feasible in all the patients including the one with pancreatic tail cancer. No procedure-related complications were documented. The median radius of pancreatic necrosis created by photodynamic therapy (PDT) was $0.85 \mathrm{~cm}$ and volume of pancreatic necrosis on CT scan at one month post-PDT was $1.9 \mathrm{~cm}^{3}$. The median follow-up period was five months and all the patients showed stable disease during the follow-up. For the patient with pancreatic tail cancer, the follow-up period was three months. Authors suggested utilization of EUS-PDT as a salvage treatment for patients with locally advanced pancreaticobiliary cancers who are poor surgical candidates and/or had progression despite chemoradiotherapy.

\section{CONCLUSIONS}

EUS has come a long way from being a diagnostic to an interventional procedure. This review of clinical studies demonstrated that there are many possibilities for EUS-guided direct interventions in solid pancreatic tumors. The EUSFNI techniques are well-developed. However, more effective and clinically proven antitumor agents are necessary for their acceptance. EUS-guided fiducial marker placement is safe, technically feasible, and can be done without fluoroscopy. Moreover, the new multi-fiducial delivery system seems promising. Currently, EUS-guided ethanol ablation of pancreatic neuroendocrine tumors is reserved for those patients who are poor surgical candidates or refuse curative resection. Multicenter controlled studies are necessary to prove the efficacy of this procedure. Further refinement of technical details, such as ethanol injection volume, optimal ethanol concentration, and assessment of treatment response, is also needed. Finally, the results of the pilot studies on EUS-guided brachytherapy, EUS-PDT, and EUS-RFA, are promising. More studies with a greater number of patients are needed to establish the role of these procedures in the treatment of pancreatic cancer and pancreatic neuroendocrine tumors.

\section{Conflicts of Interest}

$\mathrm{JH}$ has no financial conflicts of interest. $\mathrm{KJC}$ is a consultant for and receives research support and lecture fees from Cook Medical.

\section{REFERENCES}

1. Hasan MK, Hawes RH. EUS-guided FNA of solid pancreas tumors. Gastrointest Endosc Clin N Am 2012;22:155-167, vii.

2. Samarasena JB, Nakai Y, Chang KJ. Endoscopic ultrasonography-guided fine-needle aspiration of pancreatic cystic lesions: a practical approach to diagnosis and management. Gastrointest Endosc Clin N Am 2012;22:169-185, vii.

3. Giovannini M. Endoscopic ultrasonography-guided pancreatic drainage. Gastrointest Endosc Clin N Am 2012;22:221-230, viii.

4. Levy MJ, Chari ST, Wiersema MJ. Endoscopic ultrasound-guided celiac neurolysis. Gastrointest Endosc Clin N Am 2012;22:231-247, viii.

5. Iwashita T, Lee JG. Endoscopic ultrasonography-guided biliary drainage: rendezvous technique. Gastrointest Endosc Clin N Am 2012;22:249-258, viii-ix.

6. Yamao K, Hara K, Mizuno N, et al. Endoscopic ultrasound-guided choledochoduodenostomy for malignant lower biliary tract obstruction. Gastrointest Endosc Clin N Am 2012;22:259-269, ix.

7. Park DH. Endoscopic ultrasonography-guided hepaticogastrostomy. Gastrointest Endosc Clin N Am 2012;22:271-280, ix.

8. Prasad GA, Varadarajulu S. Endoscopic ultrasound-guided abscess drainage. Gastrointest Endosc Clin N Am 2012;22:281-290, ix.

9. Choi JH, Lee SS. Endoscopic ultrasonography-guided gallbladder drainage for acute cholecystitis: from evidence to practice. Dig Endosc 2015;27:1-7.

10. Weilert F, Binmoeller KF. EUS-guided vascular access and therapy. Gastrointest Endosc Clin N Am 2012;22:303-314, x.

11. Nakai Y, Chang KJ. Endoscopic ultrasound-guided antitumor agents. 
Gastrointest Endosc Clin N Am 2012;22:315-324, x.

12. Jin Z, Chang KJ. Endoscopic ultrasound-guided fiducial markers and brachytherapy. Gastrointest Endosc Clin N Am 2012;22:325-331, x.

13. Yoon WJ, Brugge WR. Endoscopic ultrasonography-guided tumor ablation. Gastrointest Endosc Clin N Am 2012;22:359-369, xi.

14. Itoi T, Binmoeller KF. EUS-guided anastomosis. Gastrointest Endosc Clin N Am 2012;22:371-377, xi.

15. Chang KJ, Samarasena JB, Iwashita T, Nakai Y, Lee JG. Endo-hepatology: a new paradigm. Gastrointest Endosc Clin N Am 2012;22:379-385, xi.

16. Huang JY, Samarasena JB, Tsujino T, et al. EUS-guided portal pressure gradient measurement with a simple novel device: a human pilot study. Gastrointest Endosc 2016 Sep 29 [Epub]. https://doi.org/10.1016/ j.gie.2016.09.026

17. Jürgensen C, Schuppan D, Neser F, Ernstberger J, Junghans U, Stölzel U. EUS-guided alcohol ablation of an insulinoma. Gastrointest Endosc 2006;63:1059-1062.

18. Muscatiello N, Salcuni A, Macarini L, et al. Treatment of a pancreatic endocrine tumor by ethanol injection guided by endoscopic ultrasound. Endoscopy 2008;40 Suppl 2:E258-E259.

19. Deprez PH, Claessens A, Borbath I, Gigot JF, Maiter D. Successful endoscopic ultrasound-guided ethanol ablation of a sporadic insulinoma. Acta Gastroenterol Belg 2008;71:333-337.

20. Vleggaar FP, Bij de Vaate EA, Valk GD, Leguit RJ, Siersema PD. Endoscopic ultrasound-guided ethanol ablation of a symptomatic sporadic insulinoma. Endoscopy 2011;43 Suppl 2 UCTN:E328-E329.

21. Levy MJ, Thompson GB, Topazian MD, Callstrom MR, Grant CS, Vella A. US-guided ethanol ablation of insulinomas: a new treatment option. Gastrointest Endosc 2012;75:200-206.

22. Qin SY, Lu XP, Jiang HX. EUS-guided ethanol ablation of insulinomas: case series and literature review. Medicine (Baltimore) 2014;93:e85.

23. Park DH, Choi JH, Oh D, et al. Endoscopic ultrasonography-guided ethanol ablation for small pancreatic neuroendocrine tumors: results of a pilot study. Clin Endosc 2015;48:158-164.

24. Chang KJ, Nguyen PT, Thompson JA, et al. Phase I clinical trial of allogeneic mixed lymphocyte culture (cytoimplant) delivered by endoscopic ultrasound-guided fine-needle injection in patients with advanced pancreatic carcinoma. Cancer 2000;88:1325-1335.

25. Levy MJ, Alberts SR, Bamlet WR, et al. EUS-guided fine-needle injection of gemcitabine for locally advanced and metastatic pancreatic cancer. Gastrointest Endosc 2016 Nov 23 [Epub]. https://doi.org/10.1016/ j.gie.2016.11.014.

26. Hecht JR, Bedford R, Abbruzzese JL, et al. A phase I/II trial of intratumoral endoscopic ultrasound injection of ONYX-015 with intravenous gemcitabine in unresectable pancreatic carcinoma. Clin Cancer Res 2003;9:555-561.

27. Hecht JR, Farrell JJ, Senzer N, et al. EUS or percutaneously guided intratumoral TNFerade biologic with 5-fluorouracil and radiotherapy for first-line treatment of locally advanced pancreatic cancer: a phase I/II study. Gastrointest Endosc 2012;75:332-338.

28. Hanna N, Ohana P, Konikoff FM, et al. Phase 1/2a, dose-escalation, safety, pharmacokinetic and preliminary efficacy study of intratumoral administration of BC-819 in patients with unresectable pancreatic cancer. Cancer Gene Ther 2012;19:374-381.

29. Irisawa A, Takagi T, Kanazawa M, et al. Endoscopic ultrasound-guided fine-needle injection of immature dendritic cells into advanced pancreatic cancer refractory to gemcitabine: a pilot study. Pancreas 2007;35:189-190.

30. Pishvaian AC, Collins B, Gagnon G, Ahlawat S, Haddad NG. EUS-guided fiducial placement for CyberKnife radiotherapy of mediastinal and abdominal malignancies. Gastrointest Endosc 2006;64:412-417.

31. Park WG, Yan BM, Schellenberg D, et al. EUS-guided gold fiducial insertion for image-guided radiation therapy of pancreatic cancer: 50 successful cases without fluoroscopy. Gastrointest Endosc 2010;71:513518.
32. Sanders MK, Moser AJ, Khalid A, et al. EUS-guided fiducial placement for stereotactic body radiotherapy in locally advanced and recurrent pancreatic cancer. Gastrointest Endosc 2010;71:1178-1184.

33. Varadarajulu S, Trevino JM, Shen S, Jacob R. The use of endoscopic ultrasound-guided gold markers in image-guided radiation therapy of pancreatic cancers: a case series. Endoscopy 2010;42:423-425.

34. Khashab MA, Kim KJ, Tryggestad EJ, et al. Comparative analysis of traditional and coiled fiducials implanted during EUS for pancreatic cancer patients receiving stereotactic body radiation therapy. Gastrointest Endosc 2012;76:962-971.

35. Draganov PV, Chavalitdhamrong D, Wagh MS. Evaluation of a new endoscopic ultrasound-guided multi-fiducial delivery system: a prospective non-survival study in a live porcine model. Dig Endosc 2013;25:615621.

36. Chavalitdhamrong D, DiMaio CJ, Siersema PD, Wagh MS. Technical advances in endoscopic ultrasound-guided fiducial placement for the treatment of pancreatic cancer. Endosc Int Open 2015;3:E373-E377.

37. Majumder S, Berzin TM, Mahadevan A, et al. Endoscopic ultrasound-guided pancreatic fiducial placement: how important is ideal fiducial geometry? Pancreas 2013;42:692-695.

38. Choi JH, Seo DW, Park DH, Lee SK, Kim MH. Fiducial placement for stereotactic body radiation therapy under only endoscopic ultrasonography guidance in pancreatic and hepatic malignancy: practical feasibility and safety. Gut Liver 2014;8:88-93.

39. Dávila Fajardo R, Lekkerkerker SJ, van der Horst A, et al. EUS-guided fiducial markers placement with a 22-gauge needle for image-guided radiation therapy in pancreatic cancer. Gastrointest Endosc 2014;79:851855.

40. Dhadham GC, Hoffe S, Harris CL, Klapman JB. Endoscopic ultrasound-guided fiducial marker placement for image-guided radiation therapy without fluoroscopy: safety and technical feasibility. Endosc Int Open 2016;4:E378-E382.

41. Law JK, Singh VK, Khashab MA, et al. Endoscopic ultrasound (EUS)-guided fiducial placement allows localization of small neuroendocrine tumors during parenchymal-sparing pancreatic surgery. Surg Endosc 2013;27:3921-3926.

42. Sun S, Xu H, Xin J, Liu J, Guo Q, Li S. Endoscopic ultrasound-guided interstitial brachytherapy of unresectable pancreatic cancer: results of a pilot trial. Endoscopy 2006;38:399-403.

43. Jin Z, Du Y, Li Z, Jiang Y, Chen J, Liu Y. Endoscopic ultrasonography-guided interstitial implantation of iodine 125 -seeds combined with chemotherapy in the treatment of unresectable pancreatic carcinoma: a prospective pilot study. Endoscopy 2008;40:314-320.

44. Sun S, Ge N, Wang S, Liu X, Wang G, Guo J. Pilot trial of endoscopic ultrasound-guided interstitial chemoradiation of UICC-T4 pancreatic cancer. Endosc Ultrasound 2012;1:41-47.

45. Facciorusso A, Maso MD, Barone M, Muscatiello N. Echoendoscopic ethanol ablation of tumor combined to celiac plexus neurolysis improved pain control in a patient with pancreatic adenocarcinoma. Endosc Ultrasound 2015;4:342-344.

46. Facciorusso A, Di Maso M, Serviddio G, Larghi A, Costamagna G, Muscatiello N. Echoendoscopic ethanol ablation of tumor combined with celiac plexus neurolysis in patients with pancreatic adenocarcinoma. J Gastroenterol Hepatol 2017;32:439-445.

47. Pai M, Habib N, Senturk H, et al. Endoscopic ultrasound guided radiofrequency ablation, for pancreatic cystic neoplasms and neuroendocrine tumors. World J Gastrointest Surg 2015;7:52-59.

48. Lakhtakia S, Ramchandani M, Galasso D, et al. EUS-guided radiofrequency ablation for management of pancreatic insulinoma by using a novel needle electrode (with videos). Gastrointest Endosc 2016;83:234239.

49. Arcidiacono PG, Carrara S, Reni M, et al. Feasibility and safety of EUS-guided cryothermal ablation in patients with locally advanced pancreatic cancer. Gastrointest Endosc 2012;76:1142-1151.

50. Song TJ, Seo DW, Lakhtakia S, et al. Initial experience of EUS-guided 
radiofrequency ablation of unresectable pancreatic cancer. Gastrointest Endosc 2016;83:440-443.

51. Chan HH, Nishioka NS, Mino M, et al. EUS-guided photodynamic therapy of the pancreas: a pilot study. Gastrointest Endosc 2004;59:9599.

52. Yusuf TE, Matthes K, Brugge WR. EUS-guided photodynamic therapy with verteporfin for ablation of normal pancreatic tissue: a pilot study in a porcine model (with video). Gastrointest Endosc 2008;67:957-961.

53. Choi JH, Oh D, Lee JH, et al. Initial human experience of endoscopic ultrasound-guided photodynamic therapy with a novel photosensitizer and a flexible laser-light catheter. Endoscopy 2015;47:1035-1038. 